Interfaces and Free Boundaries 15 (2013), 263-280

DOI $10.4171 / \mathrm{IFB} / 303$

\title{
Numerical approximation of capillary surfaces in a negative gravitational field
}

\author{
HANNE HARDERING \\ Hanne Hardering, Freie Universität Berlin, Institut für Mathematik, Arnimallee 6, 14195 Berlin, \\ Germany \\ E-mail: harderin@math.fu-berlin.de
}

[Received 28 October 2011 and in revised form 25 June 2013]

\begin{abstract}
A capillary surface in a negative gravitational field describes the shape of the surface of a hanging drop in a capillary tube with wetting material on the bottom. Mathematical modeling leads to the volume- and obstacle-constrained minimization of a nonconvex nonlinear energy functional of mean curvature type which is unbounded from below. In 1984 Huisken proved the existence and regularity of local minimizers of this energy under the condition on gravitation being sufficiently weak. We prove convergence of a first order finite element approximation of these minimizers. Numerical results demonstrating the theoretic convergence order are given.
\end{abstract}

2010 Mathematics Subject Classification: Primary 65N15, 53A10; Secondary 65N30.

Keywords: Capillary surface, obstacle problem, finite elements, discretization error bound

\section{Introduction}

Solvability of the capillarity problem in a positive gravitational field was shown in [6], regularity of such a solution in [7]. Obstacle and volume constraints were treated in [14]. In [13] it was shown that there exists a local minimizer to the capillarity problem with fixed volume and an obstacle constraint in a negative gravitational field for sufficiently weak gravitation.

The approximation of minimal surfaces by finite elements has been discussed before, i.e., in [15] for a two-dimensional surface over a convex domain.

Convergence of the approximation of a two-dimensional capillary surface in a positive gravitational field by first order finite elements was proven in [19]. The inclusion of a volume constraint was briefly addressed. Negative gravitation and obstacle constraints have not been examined in this context.

In [21] optimal rates of the convergence of the finite element method for a greater class of quasi-linear elliptic systems of second order in arbitrary dimensions were proven using continuation methods. This covers the approximation of capillary surfaces with small negative gravitation. Some arguments are quite similar to our own as they deal with possible global nonconvexity by looking at regions of convexity close to the continuous solutions. However, it is not obvious how to generalize the methods used to capillary surfaces over obstacles since they are not compatible with variational inequalities.

There is a wide variety of studies of related problems, which use discretization schemes in order to numerically compute bifurcation diagrams and study the qualitative behaviour of capillary surfaces over a range of parameters. One example concerns the shape of capillary surfaces with a free boundary (instead of surfaces over a fixed domain) studied in [22], where the analyzed problems 
are reduced to ordinary differential equations by looking at solutions over a one-dimensional domain or axisymmetric solutions over a two-dimensional domain. Another example is the shape of liquid bridges, i.e., graphs over a 2-dimensional cylinder in an arbitrary gravitational field, studied in [18]. Unfortunately, in many such studies rigorously proven discretization error estimates are still missing.

In this work we will consider $n$-dimensional capillary surfaces of fixed volume over an obstacle in negative gravitation. We will show existence of constrained minimizers of a first order finite element discretization of the energy functional as well as convergence of the discrete to the continuous solution. For $n=2$ we show that the constrained minimizers are indeed local solutions to the discrete problem.

Our method of proof provides the optimal order of approximation although the problem has global properties usually considered problematic, such as nonlinearity and nonconvexity. This is possible because we are only interested in the behavior of discrete solutions locally, i.e., near the continuous ones. We are using a concept of locality specific to the field of partial differential equations. By using Poincaré's inequality, we will exploit the higher order of the nonlinearity to establish regions of convexity for the problem where we can find unique minimizers. Modifications of the convergence proof of [19] will show that these minimizers in the restricted regions converge to the continuous solution. Using the boundedness of the continuous solution established in [13] we then show for $n=2$ that we can thus obtain a discrete solution which lies in the interior of a region of convexity and therefore is a local minimizer of the energy functional. The convergence result then implies that this discrete solution converges to the continuous one in the $W^{1,2}$-norm with linear dependence on the mesh size.

After a mathematical description of the problem we will give a short overview of previous results leading to the work of this paper. In the following main part we show existence of solutions to a finite element discretization of the capillarity problem in a negative gravitational field as well as a convergence result. We will conclude this paper by giving some numerical results illustrating the theoretic convergence order.

\section{The continuous problem}

A capillary surface is a surface of prescribed mean curvature with Neumann boundary conditions. The name is derived from the typical example of the surface of a liquid rising in a capillary tube.

The principle of energy minimization states that the surface will be in a state of minimal potential energy. In order to obtain a mathematical expression for the energy we assume that the surface $S$ of the liquid can be expressed as the graph of a function $u: \Omega \rightarrow \mathbb{R}$ over the cross section $\Omega \subseteq \mathbb{R}^{n}$ of the tube, where $\Omega$ is a connected and bounded domain. This parametrization of the surface $S=\operatorname{graph}(u)$ induces a metric $g$ on $S$ defined by

$$
g_{i j}(x)=\delta_{i j}+D_{i} u(x) D_{j} u(x),
$$

where

$$
D_{k} u=\frac{\partial u}{\partial x^{k}}, \quad k=1, \ldots, n,
$$

and $\delta_{i j}$ denotes the Kronecker symbol.

The shape of the surface is then given by a minimizer of the energy functional, i.e., the capillarity problem reads

$$
u \in K: J(u) \leqslant J(v), \quad \forall v \in K,
$$


where $K$ is a suitable function set and $J$ is given by

$$
J(v)=\int_{\Omega} \sqrt{1+|D v|^{2}} d x+\int_{\Omega} \int_{0}^{v(x)} H(x, t) d t d x+\int_{\partial \Omega} \beta v d \mathcal{H}_{n-1} .
$$

Here the first term models the cohesive energy as proportional to the area of the surface. The second term describes the gravitational energy, where $H \in C^{0,1}\left(\mathbb{R}^{n} \times \mathbb{R}\right)$ describes the gravitational potential. The third term is related to the adhesive energy at the boundary of the capillary tube.

The Euler-Lagrange equation corresponding to (1) has the form

$$
\begin{gathered}
A u+H(x, u)=0, \quad \text { in } \Omega \\
-\sum_{i=1}^{n} a^{i}(D u) \mu_{i}=\beta, \quad \text { on } \partial \Omega,
\end{gathered}
$$

where $a^{i}(D u)=\frac{D_{i} u}{\sqrt{1+|D u|^{2}}}$ and $\mu$ denotes the outer normal to $\partial \Omega$. Note that

$$
A u=-\sum_{i=1}^{n} D_{i}\left(a^{i}(D u)\right)
$$

is an expression for the mean curvature of $S$, and that the left hand side of the boundary condition (4) gives the cosine of the contact angle.

Following the work of Huisken [13] we are concerned with the capillarity problem over an obstacle in a gravitational field. For modeling we consider the surface of a liquid of fixed volume $V$ in a capillary tube. We assume that the bottom of the tube can be represented by an obstacle function $\psi$ and is of a material which is perfectly wetting, i.e., it is completely covered by a thin film of the liquid and thus does not add to the energy functional. This situation is depicted on the left hand side of Figure 1. We may also consider the liquid being in an upside down capillary tube. This is depicted on the right hand side of Figure 1. In the latter case we will then reverse the coordinate system so that we are again in the setting of a capillary surface over an obstacle.

The capillarity problem is given by (1) with

$$
K=W^{1, \infty}(\Omega) \cap\{v \geqslant \psi\} \cap\left\{\int_{\Omega}(v-\psi) d x=V\right\},
$$

and a gravitational potential of the form

$$
H(x, t)=-\kappa t,
$$

where $-\kappa>0$ in the case of a "sitting" liquid, and $-\kappa<0$ in the case of a "hanging" liquid. The focus of this work is the setting in negative gravitation, i.e. $-\kappa<0$. Note that in [13] Huisken considered a more general gravitational potential of the form $H(x, t)=-\kappa t+\tilde{H}(x, t)$ with $\frac{\partial \tilde{H}}{\partial t}>0$. The above approximation (6) of the gravitational field as constant is possible in many physically important cases like gravity on Earth. Generalizations of our method to other applications like centrifuges should be possible (using suitable cut-offs) but rather technical.

We assume furthermore $\beta \in C^{0,1}(\partial \Omega)$ with

$$
|\beta| \leqslant 1-a, \quad a>0 .
$$




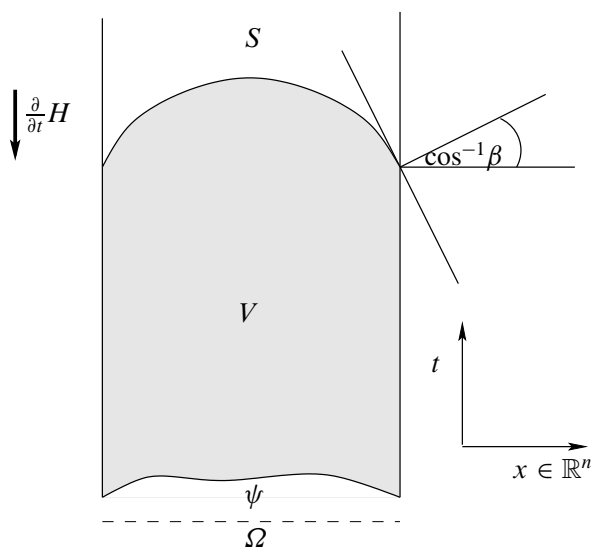

(a) Positive gravitation

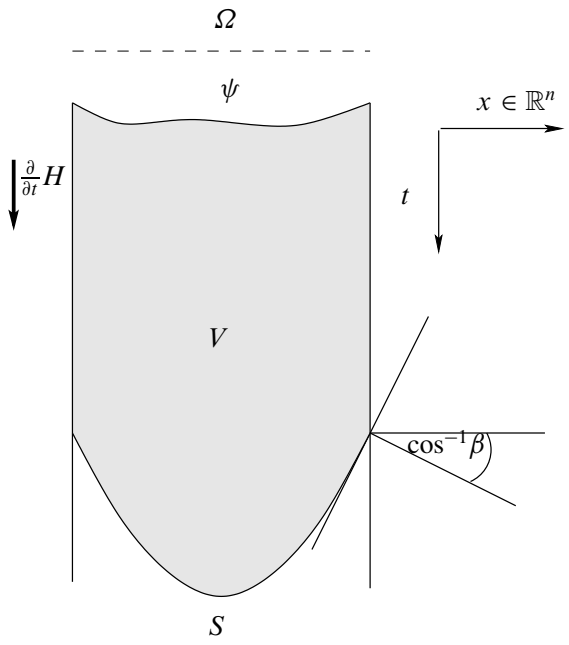

(b) Negative gravitation

FIG. 1. Liquid in a capillary tube

To motivate the last condition, note that $\beta \geqslant 1$ means that the liquid will be in a state of lesser energy if it pulls back from the tube (lotus effect), and $\beta \leqslant-1$ means that the material of the tube is perfectly wetting (just as the obstacles considered in this work). Our model will not account for such situations.

Because of the negative quadratic term the energy functional $J$ defined by (2) with (6) may be neither convex nor bounded from below. This can be easily seen in the following example.

EXAMPLe Assume $\psi \equiv 0$ and $V=1$. Consider the mollifier functions

$$
\eta_{\epsilon}:=\frac{1}{\epsilon^{n}} \eta\left(\frac{x}{\epsilon}\right)
$$

for $\epsilon>0$ on the unit ball $\Omega=B_{1}(0) \subset \mathbb{R}^{n}$ for $n \geqslant 2$ (cf. [4]). The standard mollifier $\eta$ is defined by

$$
\eta(x):=C \exp \left(\frac{1}{|x|^{2}-1}\right),
$$

where the constant $C>0$ is chosen such that $\int_{\Omega} \eta d x=1$. Hence, $\eta_{\epsilon} \in K$, where $K$ is defined by (5), and $J\left(\eta_{\epsilon}\right) \rightarrow-\infty$ as $\epsilon \rightarrow 0$.

Thus, we cannot generally expect the minimization problem (1) to have global minimizers. Nevertheless, we can study local minimizers which are solutions to the corresponding variational inequality

$$
\int_{\Omega} \frac{D u \cdot D(v-u)}{\sqrt{1+|D u|^{2}}} d x-\kappa \int_{\Omega} u(v-u) d x+\int_{\partial \Omega} \beta(v-u) d \mathcal{H}_{n-1} \geqslant 0, \quad \forall v \in K .
$$

Even in the context of positive gravitational fields, i.e., $\frac{\partial H}{\partial t}>0$, we cannot expect the existence of 
a bounded capillary surfaces if $\partial \Omega$ has vertices (cf. [5]). Thus, we will assume that $\partial \Omega$ is of class $C^{2, \alpha}$.

Various results on the well-posedness of capillarity problems can be found in the literature. Relying solely on $B V$-techniques Gerhardt proved in [6] existence and uniqueness of solutions to (1) without a volume or obstacle constraint in a positive gravitational field, i.e., under the assumption $\frac{\partial H}{\partial t}>0$. Using a different approach, he showed the following global regularity result in [7].

TheOREM 2.1 Let $\partial \Omega \in C^{2, \alpha}, H, \beta \in C^{1, \alpha}$ and $\frac{\partial H}{\partial t}>0$. The capillarity problem (1) has a unique solution $u \in C^{2, \vartheta}(\Omega)$, where $\vartheta, 0<\vartheta<1$, is determined by $H, \beta$, and $\Omega$.

The proof relies on a rather technical a priori estimate for the gradient (cf. [14, Section 2]). The proof itself is done by a method of continuity and uses standard theory of uniformly elliptic differential equations (cf. [17], [8, Thm. 17.30]).

Relying on Theorem 2.1 Huisken extended the theory to the capillarity problem with an obstacle constraint in a positive gravitational field [14], and to the capillarity problem in a negative gravitational field [13]. For the latter case he showed that for sufficiently weak gravity, i.e., sufficiently small $\kappa>0$, the variational inequality (8) admits a solution.

Theorem 2.2 Let $\partial \Omega$ be of the class $C^{2, \alpha}, \psi \in C^{2}(\bar{\Omega})$ and $H, \beta \in C^{1, \alpha}$ with the properties (6) and (7). There exists a $\kappa_{0}$ such that for $0<\kappa<\kappa_{0}$ the following applies:

1. The capillarity problem in a negative gravitational field (8) admits a solution

$$
u \in W^{1, \infty}(\Omega) \cap W^{2,2}(\Omega) \cap W_{l o c}^{2, \infty}(\Omega)
$$

with continuous tangential derivatives at the boundary.

2. If $n=2$ then $u \in C^{1}(\bar{\Omega})$.

3. If we assume that $\partial \Omega$ is of class $C^{3, \alpha}, \beta \in C^{1,1}(\partial \Omega)$, and $\psi$ satisfies

$$
-\frac{D^{i} \psi}{\sqrt{1+|D \psi|^{2}}} \mu_{i} \geqslant \beta, \quad \text { on } \partial \Omega
$$

then

$$
u \in W^{2, \infty}(\Omega) .
$$

4. For $\kappa$ small enough the solution $u$ is is unique in the class of functions satisfying $\|u\|_{C^{1}(\Omega)} \leqslant M$.

\section{Discretization}

In order to give a numerical approximation of capillary surfaces we will employ a first order finite element method which is also used by Mittelmann for the case of positive gravitational fields [19]. We will extend his results to the case of a negative gravitational field, where $J$ is non-convex.

For each $h, 0<h<h_{0}$, let $\Omega_{h}=\bigcup_{j=1}^{L(h)} T_{j}$ be a finite collection of $n$-simplices with disjoint interiors such that each face of a simplex is either the face of another simplex or has its vertices on $\partial \Omega$. We assume the triangulation to be shape regular in the sense that each simplex is contained in a ball of radius $h$ and contains a ball of radius $\gamma h$ for a fixed $0<\gamma<1$. Since in general $\Omega_{h} \not \subset \Omega$ we assume that any solution $u$ of the capillarity problem (8) may be extended to a domain $\tilde{\Omega} \subset \mathbb{R}^{n}$ with $\bar{\Omega} \subset \tilde{\Omega}$ and $\Omega_{h} \subset \tilde{\Omega}$, such that the extension is of the same class as $u$, coincides with $u$ in $\Omega$, and the extension operator is continuous. Existence of such an operator was shown in [20, Ch. 2]. For simplicity the extension will again be denoted by $u$. 
Let $S_{h}$ denote the space of linear finite elements on $\tilde{\Omega}$, i.e., $S_{h}=\left\{v \in C(\tilde{\Omega}):\left.v\right|_{T_{j}}\right.$ is linear, $j=1, \ldots, L(h)$, and piecewise linearly extended outside $\left.\Omega_{h}\right\}$. The finite element space $S_{h}$ is spanned by the nodal basis

$$
\Lambda_{h}:=\left\{\lambda_{p} \in S_{h} \mid p \in N_{h}\right\}, \quad \lambda_{p}(q)=\delta_{p q} \forall p, q \in N_{h},
$$

where $N_{h}$ denotes the set of all vertices corresponding to the triangulation $\Omega_{h}$. For every continuous function $v: \Omega \rightarrow \mathbb{R}$ we define its interpolation $v_{I} \in S_{h}$ by

$$
v_{I}(x):=\sum_{p \in N_{h}} v(p) \lambda_{p}(x) .
$$

For the interpolation error the following estimates hold [2].

THeOREM 3.1 Let $h$ be sufficiently small, and $u \in W^{k+1, p}(\Omega), k \geqslant 0,1 \leqslant p \leqslant \infty$ with $(k+1) p>n$. Then there exists a constant $C_{1}$ such that the linear interpolation error for $m=0,1$ can be estimated by

$$
\left|u-u_{I}\right|_{m, p, \Omega_{h}} \leqslant C_{1} \gamma^{-m} h^{k+1-m}|u|_{k+1, p, \Omega_{h}} .
$$

Here and below

$$
\begin{aligned}
|v|_{k, p, \Omega} & =\left(\sum_{|\alpha|=k} \int_{\Omega}\left|D^{\alpha} v\right|^{p} d x\right)^{\frac{1}{p}}, \\
\|v\|_{k, p, \Omega} & =\sum_{l \leqslant k}|v|_{l, p, \Omega}
\end{aligned}
$$

denote the Sobolev (semi) norms for $k \in \mathbb{N}$ and $1 \leqslant p \leqslant \infty$ with the usual modification when $p=\infty$. If $k=0$ this index may be omitted.

In the following we will assume that the setting is such that Theorem 2.2 (3) holds, i.e., that there exists a solution

$$
u \in W^{2, \infty}(\tilde{\Omega})
$$

to (8) where $H$ is given by (6) and $K$ is defined by (5). Note that Morrey's inequality (cf. e.g. [4]) implies for $p>n$ that

$$
W^{1, p}(\Omega) \subset C^{0,1-\frac{n}{p}}(\Omega) .
$$

Therefore, interpolation is well defined for $u \in W^{1, \infty}(\Omega)$.

For $n \geqslant 4$ we will especially need the higher regularity assumptions of Theorem 2.2 (3) in order to employ Theorem 3.1 to estimate the interpolation error by

$$
\left|u-u_{I}\right|_{0,2, \Omega_{h}} \leqslant C h^{2},
$$

where the constant depends on $|u|_{2, \infty, \tilde{\Omega}}$.

REMARK 3.2 The case $n \geqslant 3$ is often covered only implicitly in related works like [15] and [19]. The restriction on the dimension in Theorem 3.1 can be overcome by the stronger regularity assumption on the continuous solution. This generalization works similarly in [15] and [19]. 
Let $\psi_{I}$ denote the linear interpolation of the obstacle function $\psi$. Let furthermore

$$
V^{h}:=V-\int_{\Omega_{h}} \psi_{I} d x+\int_{\Omega} \psi d x
$$

denote the discrete prescribed volume. We approximate the function set $K$ by

$$
K_{h}:=S_{h} \cap\left\{v_{h} \geqslant \psi_{I}\right\} \cap\left\{\int_{\Omega_{h}}\left(v_{h}-\psi_{I}\right) d x=V^{h}\right\} .
$$

The discrete energy functional is defined by

$$
J_{h}\left(v_{h}\right):=-\frac{\kappa}{2} a_{h}\left(v_{h}, v_{h}\right)-l_{h}\left(v_{h}\right)+\phi_{h}\left(v_{h}\right),
$$

with

$$
\begin{aligned}
a_{h}\left(v_{h}, w_{h}\right) & =\int_{\Omega_{h}} v_{h} w_{h} d x, \\
l_{h}\left(v_{h}\right) & =-\int_{\partial \Omega_{h}} \beta_{I} v_{h} d s_{h}, \\
\phi_{h}\left(v_{h}\right) & =\int_{\Omega_{h}} \sqrt{1+\left|D v_{h}\right|^{2}} d x,
\end{aligned}
$$

for $v_{h}, w_{h} \in K_{h}$. The choice of the sign of $\kappa$ depends on the direction of the gravitational force, i.e., $\kappa>0$ for negative gravitation, $\kappa<0$ for positive gravitation. Note that we can view $\partial \Omega_{h}$ as a triangulation of $\partial \Omega$ and that $\beta \in C^{0,1}(\partial \Omega)$ implies that the finite element interpolation $\beta_{I}$ on the boundary is well-defined.

As in the continuous case, we cannot expect a solution to the global minimization problem

$$
u_{h} \in K_{h}: J_{h}\left(u_{h}\right) \leqslant J_{h}\left(v_{h}\right) \quad \forall v_{h} \in K_{h},
$$

to exist for general $\kappa>0$ because of the non-convex term $-\frac{\kappa}{2} \int_{\Omega_{h}} v_{h}^{2} d x$. We consider instead the corresponding variational inequality for critical points (we concentrate on local minima)

$$
\int_{\Omega_{h}} \frac{D u_{h} \cdot D\left(v_{h}-u_{h}\right)}{\sqrt{1+\left|D u_{h}\right|^{2}}} d x-\kappa a_{h}\left(u_{h}, v_{h}-u_{h}\right)-l_{h}\left(v_{h}-u_{h}\right) \geqslant 0, \quad \forall v_{h} \in K_{h} .
$$

In the case of positive gravitation, i.e., for the problem (12) with $\kappa<0$ and $K_{h}=S_{h}$, uniqueness and existence of solutions follow by the direct method of the calculus of variations (cf., e.g., [16]). In [19] the following convergence result for the difference of the discrete solution $u_{h}$ and the interpolation $u_{I}$ of the continuous solution is proven.

THEOREM 3.3 Let $\Omega \subset \mathbb{R}^{2}$ be a bounded domain with $\partial \Omega \in C^{2}$. If the continuous problem (1) without an obstacle bound and $H(x, t)=\kappa t$ has a solution $u \in W^{2,2}(\tilde{\Omega}) \cap W^{1, \infty}(\tilde{\Omega})$, then

$$
\left\|u_{h}-u_{I}\right\|_{1,2, \Omega_{h}} \leqslant C_{2} h,
$$

for all $0<h<h_{0}, h_{0}$ sufficiently small, where the constant $C_{2}$ is independent of $h$. 
In the following we will need two lemmas used also in [19] to analyze the variational crimes due to the difference between $\Omega$ and $\Omega_{h}$ and their boundaries. We denote

$$
\omega:=\left(\Omega-\Omega_{h}\right) \cup\left(\Omega_{h}-\Omega\right) .
$$

LEMMA 3.4 For sufficiently small $h$ there exists a constant $C_{3}=C_{3}(\Omega)$ such that we may estimate for $v_{h} \in S_{h}$

$$
\left\|v_{h}\right\|_{1, \omega} \leqslant C_{3} h^{2}\left\|v_{h}\right\|_{1,1, \Omega_{h}} .
$$

LEMmA 3.5 For sufficiently small $h$ there exists a constant $C_{4}=C_{4}(\gamma)$ such that for any function $v_{h} \in S_{h}$

$$
\left|v_{h}\right|_{1,1, \omega} \leqslant C_{4} h\left|v_{h}\right|_{1,1, \Omega_{h}} .
$$

Furthermore, we can estimate

$$
\left|l\left(v_{h}\right)-l_{h}\left(v_{h}\right)\right| \leqslant C_{5} h\left\|v_{h}\right\|_{1,1, \Omega_{h}},
$$

where $C_{5}$ depends on $\partial \Omega,\|\beta\|_{1, \infty}$, and $\gamma$.

Proof. The proofs of Lemma 3.4 and the first part of Lemma 3.5 rely on the fact that the distance of $\partial \Omega$ and $\partial \Omega_{h}$ is in $O\left(h^{2}\right)$ and that $D v_{h}$ is piecewise constant. They can be found in [19]. The proof of the second part of Lemma 3.5 in [19] does not directly transfer to arbitrary $n$ and does not include a $\beta$ which is not constant. However, we may salvage the proof by some minor changes.

Let $\partial \Omega_{h}$ be parametrized over $\partial \Omega$ by $\bar{x}=x+\phi(x) \nu(x)$. Then

$$
\begin{aligned}
& \left|l\left(v_{h}\right)-l_{h}\left(v_{h}\right)\right| \leqslant \mid \int_{\partial \Omega}\left(\beta_{I}(\bar{x}) \mu_{h}(x)\left(v_{h}(x)-v_{h}(\bar{x})\right)\right) \\
& +\left(\beta(x)-\beta_{I}(\bar{x})\right) v_{h}(x)+\beta_{I}(\bar{x})\left(1-\mu_{h}(x)\right) v_{h}(x) d s \mid,
\end{aligned}
$$

where $\mu_{h} d s=d s_{h}$. Note that $\left\|\mu_{h}-1\right\|_{\infty, \partial \Omega} \leqslant C h$ (cf. e.g. [3]). Thus we may estimate

$$
\begin{aligned}
\left|l\left(v_{h}\right)-l_{h}\left(v_{h}\right)\right| & \leqslant C \int_{\omega}\left|D v_{h}(x)\right| d x+C h \int_{\partial \Omega}\left|v_{h}(x)\right| d s \\
& \leqslant C h\left\|v_{h}\right\|_{1,1, \tilde{\Omega}},
\end{aligned}
$$

where we estimated the second term by a lemma proven in [6]. Using (15) and Lemma 3.4 the assertion follows.

\section{Discretization of capillary surfaces in a negative gravitational field}

Our main result is the following:

THEOREM 4.1 For $n=2$ there exists a $\tilde{\kappa}$ depending on $V, \beta, \Omega$, and $\psi$ such that for $0<\kappa<\tilde{\kappa}$ and for $0<h<h_{0}$ there exists a solution to the discrete capillarity problem in a negative gravitational field with an obstacle, i.e., a function $u_{h} \in K_{h}$ satisfying (13). Furthermore, the discretization error is bounded by

$$
\left\|u_{h}-u\right\|_{1,2, \Omega_{h}} \leqslant C h .
$$


The theorem will be proven in several steps. First we prove the existence of constrained minimizers. We then discuss the convergence of these to the continuous solution. The proof of Theorem 4.1 is then given in Section 4.3.

Note that the restriction to $n=2$ is only necessary to show that the constrained minimizers are indeed local solutions to the minimization problem. For arbitrary dimension we still obtain convergence of the constrained minimizers to the continuous solution. However, this convergence will depend on the constraint.

\subsection{Existence of constrained minimizers}

Before we can prove existence of solutions to (13) we will prove the following:

Lemma 4.2 Let $M \in \mathbb{R}$. There exists $\kappa_{1}(n, \Omega, M)$ such that for $0<\kappa<\kappa_{1}$ there exists a unique solution $u_{M}$ to the discrete problem

$$
u_{M} \in K_{M}: J_{h}\left(u_{M}\right) \leqslant J_{h}(v), \quad \forall v \in K_{M},
$$

where $J_{h}$ is defined by (11) and

$$
K_{M}:=K_{h} \cap\left\{|D v|_{\infty, \Omega_{h}} \leqslant M\right\} .
$$

Proof. Note that $K_{M}$ is compact. If we can choose $\kappa$ small enough such that the energy functional $J_{h}$ is strictly convex we can apply the direct method of the calculus of variations (cf., e.g., [16]).

Let $v, w \in S_{h}$ be in the set of admissible functions $K_{M}$, i.e., we assume

$$
\int_{\Omega_{h}}\left(v-\psi_{I}\right) d x=\int_{\Omega_{h}}\left(w-\psi_{I}\right) d x=V^{h}, \quad\|D v\|_{\infty, \Omega_{h}},\|D w\|_{\infty, \Omega_{h}} \leqslant M, \quad v, w \geqslant \psi_{I} .
$$

The nonlinearity $\phi_{h}$ can be written as

$$
\begin{aligned}
\phi_{h}(v) & =\int_{\Omega_{h}} \psi(D v) d x, \\
\psi(x) & =\sqrt{1+|x|^{2}} .
\end{aligned}
$$

$\phi_{h}$ is strongly convex, i.e., we have for $\omega \in(0,1)$

$$
\phi_{h}(\omega v+(1-\omega) w) \leqslant \omega \phi_{h}(v)+(1-\omega) \phi_{h}(w)-\frac{1}{2} m \omega(1-\omega)\|D(v-w)\|_{2, \Omega_{h}}^{2},
$$

where the parameter $m$ is a lower bound on the least eigenvalue of $D^{2} \psi(D v)$. In particular we may set

$$
m=\frac{1}{\left(1+M^{2}\right)^{\frac{3}{2}}} .
$$

This yields the estimate

$$
\begin{aligned}
& J_{h}(\omega v+(1-\omega) w)-\omega J_{h}(v)-(1-\omega) J_{h}(w) \\
& \quad \leqslant \frac{1}{2} \omega(1-\omega)\left(\kappa\|v-w\|_{2, \Omega_{h}}^{2}-\frac{1}{2\left(1+M^{2}\right)^{\frac{3}{2}}}\|D(v-w)\|_{2, \Omega_{h}}^{2}\right) .
\end{aligned}
$$


Using Poincaré's inequality

$$
\left\|f-\frac{1}{\left|\Omega_{h}\right|} \int_{\Omega_{h}} f d x\right\|_{p, \Omega_{h}} \leqslant C_{6}(p, n, \Omega)\|D f\|_{p, \Omega_{h}}
$$

for $p=2$ and $f=v-w$, we see that $J_{h}$ is strictly convex if we choose

$$
\kappa<\kappa_{1}:=\frac{1}{2 C_{6}^{2}\left(1+M^{2}\right)^{\frac{3}{2}}} .
$$

For admissible functions $v \in K_{M}$ the bound on the gradient combined with (19) also implies

$$
\|v\|_{p, \Omega_{h}} \leqslant C_{6}\|D v\|_{p, \Omega_{h}}+\frac{\left|V+\int_{\Omega} \psi d x\right|}{\left|\Omega_{h}\right|^{1-\frac{1}{p}}} \leqslant C_{6}\left|\Omega_{h}\right|^{\frac{1}{p}}\left(M+\frac{\left|V+\int_{\Omega} \psi d x\right|}{\left|\Omega_{h}\right|}\right)
$$

for any $p$.

Thus, the energy functional is bounded from below. Since we are considering the minimization on the compact set $K_{M}$ this is enough to ensure the existence of a unique minimizer.

Note that the restriction of the function set to $K_{M}$ is similar to the approach in [13] where the existence of a solution was then obtained by a fixed point argument combined with a priori bounds.

\subsection{Convergence}

In this section we will extend the convergence proof for capillary surfaces in positive gravitational fields as stated in Theorem 3.3 and [19] to the $K_{M}$-bounded solutions $u_{M}$ (cf. Lemma 4.2).

Throughout the section we will use the abbreviations

$$
W:=\sqrt{1+|D u|^{2}}, \quad \text { and } \quad W_{M}:=\sqrt{1+\left|D u_{M}\right|^{2}} .
$$

We will need a priori bounds for $u_{M}$. However, we need to make sure that they do not depend on $\kappa^{-1}$. Following the approach of [19] will not yield this independence. Instead, we impose the a priori bounds by force, i.e., we restrict the function set $K_{M}$ further by setting

$$
V_{M}:=K_{M} \cap\left\{\|v\|_{2, \Omega_{h}} \leqslant\left\|u_{I}\right\|_{2, \Omega_{h}}+1\right\} \cap\left\{\|v\|_{1,1, \Omega_{h}} \leqslant\left\|u_{I}\right\|_{1,1, \Omega_{h}}+1\right\} .
$$

Note that this will not influence the solvability result in Lemma 4.2 since $V_{M}$ is a closed convex subset of $K_{M}$.

The solution to the corresponding minimization problem, i.e., (18) with $K_{M}$ replaced by $V_{M}$, will again be denoted by $u_{M}$.

Theorem 4.3 Let $M>\left\|D u_{I}\right\|_{\infty, \Omega_{h}}+1$ be large. Then there exists a $\kappa_{2}(\Omega, M, u)$ such that for $0<\kappa<\min \left\{\kappa_{1}, \kappa_{2}\right\}$

$$
\left\|u_{M}-u_{I}\right\|_{1,2, \Omega_{h}} \leqslant C h\left|W_{M}\right|_{\infty, \Omega_{h}}^{\frac{1}{2}} \leqslant C h \sqrt{M}
$$

for all $0<h<h_{0}, h_{0}$ sufficiently small, where $u_{M} \in V_{M}$ is a solution to (18) and $u_{I}$ denotes the interpolation of the continuous solution. Furthermore, for $n=2$ we have

$$
\left|u_{M}\right|_{1, \infty, \Omega_{h}} \leqslant C .
$$

The constants denoted by $C$ and $h_{0}$ depend on $V, \beta, \Omega, \psi$, and the continuous solution $u$, but not on $M, \kappa$, or $h$. 
Proof. We will proceed similarly to the proof of Theorem 3.3 in [19].

Set $e_{h}:=u_{I}-u_{M}$ and consider

$$
A^{2}:=\int_{\Omega_{h}} \frac{\left|D e_{h}\right|^{2}}{W_{M}} d x .
$$

Note that

$$
\begin{aligned}
A^{2}=\int_{\Omega} & \frac{D e_{h} \cdot D u}{W} d x-\int_{\Omega_{h}} \frac{D e_{h} \cdot D u_{M}}{W_{M}} d x \\
& +\int_{\Omega_{h}} \frac{D e_{h} \cdot D\left(u_{I}-u\right)}{W_{M}} d x \\
& +\int_{\Omega_{h}} D e_{h} \cdot D u\left(\frac{1}{W_{M}}-\frac{1}{W}\right) d x \\
& +\int_{\Omega_{h}-\Omega} \frac{D e_{h} \cdot D u}{W} d x-\int_{\Omega-\Omega_{h}} \frac{D e_{h} \cdot D u}{W} d x .
\end{aligned}
$$

We want to estimate all terms on the right hand side to obtain an inequality of the form $A^{2} \leqslant C h^{2}$. To estimate the first two terms we will use the variational formulations of the continuous and the discrete problem. The main difference to [19] is that instead of proving a priori estimates on the discrete solution we need to choose test functions fulfilling the additional bounds on the function set. This will lead to extra terms which will turn out to be of order $h^{2}$ and thus do not alter the convergence result. For completeness we will also carry out the estimates for the remaining terms which can also be found, e.g., in [19] and [2].

To bound the first term we insert the test function

$$
v:=v_{\psi}+C_{V}\left(v_{\psi}-\psi\right)
$$

into the continuous variational inequality (8), where

$$
\begin{aligned}
v_{\psi} & :=u+u_{M}-u_{I}+C_{\psi}, \\
C_{\psi} & :=\left\|\psi_{I}-\psi\right\|_{\infty, \tilde{\Omega}}+\left\|u-u_{I}\right\|_{\infty, \tilde{\Omega}}, \\
C_{V} & :=\frac{\theta}{V-\theta}, \\
\theta & :=\int_{\Omega} u_{I}-u d x+\int_{\Omega_{h}-\Omega} u_{M} d x-\int_{\Omega-\Omega_{h}} u_{M} d x-C_{\psi}|\Omega| .
\end{aligned}
$$

$C_{\psi}$ ensures that $v_{\psi}$ lies above the obstacle. $C_{V}$ enforces the continuous volume constraint. By Theorem 3.1 and Lemma 3.4 we may estimate $|\theta| \leqslant C(n, u, \Omega) h^{2}$ and thus $\left|C_{V}\right| \leqslant$ $C(n, u, \Omega, V, \psi) h^{2}<1$ for $h_{0}$ small enough. Therefore $v$ is an admissible test function for the continuous problem. The variational inequality (8) then reads

$$
\begin{aligned}
& \int_{\Omega} \frac{D e_{h} \cdot D u}{W} d x \leqslant \kappa a\left(u, e_{h}\right)+l\left(e_{h}\right) \\
&+C_{V}\left\|v_{\psi}-\psi\right\|_{1,1, \Omega}-\kappa a\left(u, C_{\psi}+C_{V}\left(v_{\psi}-\psi\right)\right)-l\left(C_{\psi}+C_{V}\left(v_{\psi}-\psi\right)\right),
\end{aligned}
$$


where $a(\cdot, \cdot)$ and $l(\cdot)$ are the continuous analoga to $a_{h}(\cdot, \cdot)$ and $l_{h}(\cdot)$.

Furthermore

$$
\begin{aligned}
\left\|v_{\psi}-\psi\right\|_{2, \Omega_{h}} & \leqslant C, \\
\left\|v_{\psi}-\psi\right\|_{1,1, \Omega_{h}} & \leqslant C
\end{aligned}
$$

with constants depending on $u, \psi$, and $\Omega$, since all parts of $v_{\psi}$ fulfill these bounds. By Theorem 3.1 we have $C_{\psi} \leqslant C h^{2}$ since we assume $\psi, u \in W^{2, \infty}$.

The variational inequality then reads

$$
\int_{\Omega} \frac{D u \cdot D e_{h}}{W} d x \leqslant \kappa a\left(u, e_{h}\right)+l\left(e_{h}\right)+C h^{2},
$$

where the constant depends on $V, \beta, \Omega, \psi$, and $u$. For the second term we insert the test function

$$
v_{h}:=u_{I}+C_{V^{h}}\left(u_{I}-\psi_{I}\right)
$$

into the discrete variational inequality (13), where

$$
\begin{aligned}
C_{V^{h}} & :=\frac{\theta_{h}}{V^{h}-\theta_{h}}, \\
\theta_{h} & :=\int_{\Omega_{h}} u-u_{I} d x+\int_{\Omega-\Omega_{h}} u d x-\int_{\Omega_{h}-\Omega} u d x .
\end{aligned}
$$

Note that $u_{I}$ does not violate the discrete obstacle constraint. $C_{V}$ enforces compliance with the volume constraint. By Theorem 3.1 and Lemma 3.4, we have $\left|\theta_{h}\right| \leqslant C h^{2}$. Thus choosing $h_{0}$ small enough depending on $n, u, \Omega, \psi$, and $V$ implies $\left|C_{V^{h}}\right| \leqslant C h^{2} \leqslant 1$. Therefore $v_{h}$ does not violate the obstacle constraint. Note furthermore that

$$
\left\|u_{I}-\psi_{I}\right\|_{p, q, \Omega_{h}} \leqslant C(u, \psi)
$$

for $(p, q) \in\{(1, \infty),(1,1),(0,2)\}$. Thus, in these Sobolev norms we can estimate

$$
\left\|v_{h}\right\|_{p, q, \Omega_{h}} \leqslant\left\|u_{I}\right\|_{p, q, \Omega_{h}}+C h^{2} \leqslant\left\|u_{I}\right\|_{p, q, \Omega_{h}}+1
$$

for $h_{0}$ small enough. This implies $v_{h} \in V_{M}$ for $M>\left\|D u_{I}\right\|_{\infty, \Omega_{h}}+1$. The variational inequality (13) for $v_{h}$ then reads

$$
\begin{aligned}
-\int_{\Omega_{h}} \frac{D u_{M} \cdot D e_{h}}{W_{M}} d x \leqslant-\kappa a_{h}\left(u_{M}, e_{h}\right)-l_{h}\left(e_{h}\right) \\
\quad+C_{V^{h}}\left(\int_{\Omega_{h}} \frac{D u_{M} \cdot D\left(u_{I}-\psi_{I}\right)}{W_{M}} d x-\kappa a_{h}\left(u_{M}, u_{I}-\psi_{I}\right)-l_{h}\left(u_{I}-\psi_{I}\right)\right),
\end{aligned}
$$

which implies

$$
-\int_{\Omega_{h}} \frac{D u_{M} \cdot D e_{h}}{W_{M}} d x \leqslant-\kappa a_{h}\left(u_{M}, e_{h}\right)-l_{h}\left(e_{h}\right)+C h^{2} .
$$

Adding (23) and (24), we obtain

$$
\int_{\Omega} \frac{D e_{h} \cdot D u}{W} d x-\int_{\Omega_{h}} \frac{D e_{h} \cdot D u_{M}}{W_{M}} d x \leqslant \kappa\left(a\left(u, e_{h}\right)-a_{h}\left(u_{M}, e_{h}\right)\right)+l\left(e_{h}\right)-l_{h}\left(e_{h}\right)+C h^{2} .
$$


To estimate the term $\kappa\left(a\left(u, e_{h}\right)-a_{h}\left(u_{M}, e_{h}\right)\right)$ note that

$$
a\left(u, e_{h}\right)-a_{h}\left(u_{M}, e_{h}\right)=a_{h}\left(e_{h}, e_{h}\right)+\int_{\Omega_{h}}\left(u-u_{I}\right) e_{h} d x+\int_{\Omega-\Omega_{h}} u e_{h} d x-\int_{\Omega_{h}-\Omega} u e_{h} d x
$$

can be approximated using Lemma 3.4 and Theorem 3.1 by

$$
a\left(u, e_{h}\right)-a_{h}\left(u_{M}, e_{h}\right) \leqslant a_{h}\left(e_{h}, e_{h}\right)+C h^{2}\left\|e_{h}\right\|_{2, \Omega_{h}}+C h^{2}\left\|e_{h}\right\|_{1,1, \Omega_{h}} .
$$

Lemma 3.5 yields

$$
\left|l\left(e_{h}\right)-l_{h}\left(e_{h}\right)\right| \leqslant C_{5} h\left\|e_{h}\right\|_{1,1, \Omega_{h}} .
$$

The first two terms of $A^{2}$ can thus be estimated by

$$
\begin{array}{r}
\int_{\Omega} \frac{D e_{h} \cdot D u}{W} d x-\int_{\Omega_{h}} \frac{D e_{h} \cdot D u_{M}}{W_{M}} d x \leqslant \kappa a_{h}\left(e_{h}, e_{h}\right)+C h^{2}\left(1+\left\|e_{h}\right\|_{1,1, \Omega_{h}}+\left\|e_{h}\right\|_{2, \Omega_{h}}\right) \\
+C h\left\|e_{h}\right\|_{1,1, \Omega_{h}} .
\end{array}
$$

We now need to estimate the remaining terms of the decomposition of $A^{2}$. The third term can be estimated using Hölder's inequality

$$
\begin{aligned}
\left|\int_{\Omega_{h}} \frac{D e_{h} \cdot D\left(u_{I}-u\right)}{W_{M}} d x\right| & \leqslant \int_{\Omega_{h}} \frac{\left|D e_{h}\right|}{\sqrt{W_{M}}}\left|D\left(u_{I}-u\right)\right| d x \\
& \leqslant A\left|u_{I}-u\right|_{1,2, \Omega_{h}} .
\end{aligned}
$$

Using Theorem 3.1, we obtain

$$
\left|\int_{\Omega_{h}} \frac{D e_{h} \cdot D\left(u_{I}-u\right)}{W_{M}} d x\right| \leqslant C h A .
$$

To estimate the fourth term note that

$$
\begin{aligned}
\left|\frac{1}{W_{M}}-\frac{1}{W}\right| & =\left|\frac{W^{2}-W_{M}^{2}}{W W_{M}\left(W+W_{M}\right)}\right| \\
& =\left|\frac{D\left(u-u_{M}\right) \cdot D\left(u+u_{M}\right)}{W W_{M}\left(W+W_{M}\right)}\right| \\
& \leqslant \frac{\left|D\left(u-u_{M}\right)\right|}{W W_{M}} .
\end{aligned}
$$

Hence, we can estimate using Hölder's inequality

$$
\begin{aligned}
\left|\int_{\Omega_{h}} D e_{h} \cdot D u\left(\frac{1}{W_{M}}-\frac{1}{W}\right) d x\right| & \leqslant \int_{\Omega_{h}} \frac{|D u|}{W} \frac{\left|D\left(u-u_{M}\right)\right|\left|D e_{h}\right|}{W_{M}} d x \\
& \leqslant \lambda \int_{\Omega_{h}} \frac{\left|D e_{h}\right|^{2}+\left|D\left(u-u_{I}\right)\right|\left|D e_{h}\right|}{W_{M}} d x \\
& \leqslant \lambda\left(A^{2}+A\left(\int_{\Omega_{h}} \frac{\left|D\left(u-u_{I}\right)\right|^{2}}{W_{M}} d x\right)^{\frac{1}{2}}\right) \\
& \leqslant \lambda A\left(A+\left|u-u_{I}\right|_{1,2, \Omega_{h}}\right),
\end{aligned}
$$


where $\lambda=\max _{\tilde{\Omega}} \frac{|D u|}{W}<1$. Taking Theorem 3.1 into account we can furthermore estimate

$$
\left|\int_{\Omega_{h}} D e_{h} \cdot D u\left(\frac{1}{W_{M}}-\frac{1}{W}\right) d x\right| \leqslant \lambda A(A+C h) .
$$

Using Lemma 3.5 we can estimate the last terms of $A^{2}$ by

$$
\int_{\Omega_{h}-\Omega} \frac{D e_{h} \cdot D u}{W} d x-\int_{\Omega-\Omega_{h}} \frac{D e_{h} \cdot D u}{W} d x \leqslant C h\left|e_{h}\right|_{1,1, \Omega_{h}} .
$$

Combining (25), (26), (27), and (28) yields

$$
\begin{aligned}
A^{2}-\kappa a_{h}\left(e_{h}, e_{h}\right) \leqslant \lambda A^{2}+C(1+\lambda) h A+C h^{2}\left(1+\left\|e_{h}\right\|_{1,1, \Omega_{h}}+\right. & \left.\left\|e_{h}\right\|_{2, \Omega_{h}}\right) \\
& +C h\left\|e_{h}\right\|_{1,1, \Omega_{h}} .
\end{aligned}
$$

$u_{M} \in V_{M}$ implies that $\left\|e_{h}\right\|_{1,1, \Omega_{h}}$ and $\left\|e_{h}\right\|_{2, \Omega_{h}}$ are bounded by a constant independent of $h$. Using Young's inequality we thus obtain

$$
\frac{1-\lambda}{2} A^{2}-\kappa a_{h}\left(e_{h}, e_{h}\right) \leqslant C h^{2}+C h\left\|e_{h}\right\|_{1,1, \Omega_{h}} .
$$

Note that the assumption $\left\|D u_{M}\right\|_{\infty, \Omega_{h}} \leqslant M$ implies

$$
\left\|D e_{h}\right\|_{2, \Omega_{h}}^{2} \leqslant \sqrt{1+M^{2}} A^{2} .
$$

Using Poincaré's inequality (19) and the volume constraint we obtain

$$
\begin{aligned}
\kappa a_{h}\left(e_{h}, e_{h}\right) & \leqslant 2 \kappa\left(\left\|e_{h}-\frac{1}{\left|\Omega_{h}\right|} \int_{\Omega_{h}} e_{h} d x\right\|_{2}^{2}+\left\|\frac{1}{\left|\Omega_{h}\right|} \int_{\Omega_{h}} e_{h} d x\right\|_{2}^{2}\right) \\
& \leqslant 2 \kappa\left(C_{6}^{2}\left\|D e_{h}\right\|_{2}^{2}+C h^{2}\right) \\
& \leqslant 2 \kappa\left(C_{6}^{2} \sqrt{1+M^{2}} A^{2}+C h^{2}\right) \\
& \leqslant \frac{1-\lambda}{4} A^{2}+2 \kappa C h^{2}
\end{aligned}
$$

for $\kappa$ small enough depending on $M$, i.e.,

$$
\kappa \leqslant \kappa_{2}:=\frac{1-\lambda}{8 C_{6}^{2} \sqrt{1+M^{2}}} .
$$

Inserting this into (30) yields

$$
A^{2} \leqslant C h^{2}+C h\left\|e_{h}\right\|_{1,1, \Omega_{h}} .
$$

Note that by Poincaré's inequality and the volume constraint we may estimate

$$
\left\|e_{h}\right\|_{1,1, \Omega_{h}} \leqslant C\left|e_{h}\right|_{1,1, \Omega_{h}}+C h^{2} .
$$


Furthermore, by Hölder's inequality we have

$$
\left|e_{h}\right|_{1,1, \Omega_{h}} \leqslant\left(\int_{\Omega_{h}} W_{M} d x\right)^{\frac{1}{2}} A \leqslant\left(\left\|u_{I}\right\|_{1,1, \Omega_{h}}+1+\left|\Omega_{h}\right|\right)^{\frac{1}{2}} A .
$$

Using Young's inequality we obtain

$$
A^{2} \leqslant C h^{2} .
$$

The desired estimate (21) than follows by

$$
\left|e_{h}\right|_{1,2, \Omega_{h}}^{2} \leqslant \sqrt{1+\left|D u_{M}\right|_{\infty, \Omega_{h}}^{2}} A^{2}
$$

and Poincaré's inequality.

For $n=2$ we will now use (30) to show that $\left|D u_{M}\right|_{\infty, \Omega_{h}}$ is bounded independent of $M$ and $h$.

For any triangle $T_{j}$ Theorem 3.1 and (32) yield

$$
\begin{aligned}
\int_{T_{j}} \frac{\left|D u_{M}\right|^{2}}{W_{M}} d x & \leqslant 2\left(A^{2}+\int_{T_{j}} \frac{\left|D u_{I}\right|^{2}}{W_{M}} d x\right) \\
& \leqslant C h^{2}+C|u|_{1, \infty, \Omega}^{2}\left|T_{j}\right| .
\end{aligned}
$$

Since $D u_{M}$ is constant on each triangle $T_{j}$ we obtain

$$
\left|D u_{M \mid T_{j}}\right| \leqslant \frac{\left|D u_{M \mid T_{j}}\right|^{2}}{\sqrt{1+\left.\left|D u_{M}\right| T_{j}\right|^{2}}}+1 \leqslant \frac{1}{\left|T_{j}\right|} \int_{T_{j}} \frac{\left|D u_{M}\right|^{2}}{W_{M}} d x+1 \leqslant C \frac{h^{2}}{\left|T_{j}\right|}+C .
$$

For $n=2$ this implies due to the shape regularity of the triangulation

$$
\left|D u_{M}\right|_{\infty, \Omega_{h}} \leqslant C .
$$

\subsection{Proof of Theorem 4.1}

We will now prove Theorem 4.1.

For $M \in \mathbb{R}$ Lemma 4.2 provides the existence of a unique solution $u_{M}$ to (18) in $K_{M}$. If we can choose $\tilde{M} \in \mathbb{R}$ such that $\left\|D u_{\tilde{M}}\right\|<\tilde{M}$, then we can find an $\epsilon>0$ such that $u_{\tilde{M}+\epsilon}=u_{\tilde{M}}$, i.e., $u_{\tilde{M}}$ is a local minimizer of $J_{h}$ in $K_{h}$, and hence a solution to the variational inequality (13).

We will use Theorem 4.3 to show the existence of such an $\tilde{M}$. By choosing $\tilde{M}$ larger than the constant in (22), we obtain

$$
\left|D u_{\tilde{M}}\right|_{\infty, \Omega}<\tilde{M}
$$

for the unique solution $u_{\tilde{M}} \in V_{\tilde{M}}$. Choosing $h_{0}$ small enough (21) provides

$$
\begin{aligned}
& \left\|u_{I}-u_{M}\right\|_{0,2, \Omega_{h}} \leqslant C h<1, \\
& \left\|u_{I}-u_{M}\right\|_{1,1, \Omega_{h}} \leqslant C h<1 .
\end{aligned}
$$


Thus $u_{\tilde{M}}$ indeed stays away from the bounds imposed by restricting to $V_{\tilde{M}}$ and is a local minimizer of $J_{h}$ in $K_{h}$. The convergence (17) follows directly from Theorem 4.3.

Note that although the constants in Theorem 4.3 and hence $h_{0}$ and $\tilde{M}$ depend on the continuous solution $u$ which itself depends on $\kappa$, this is not a circular argument, because we can assume that $u$ is bounded by a constant for all $\kappa$ smaller than some $\kappa_{0}$ as proven in [13]. This concludes the proof for $0<\kappa<\min \left\{\kappa_{0}, \kappa_{1}, \kappa_{2}\right\}$.

\section{Numerical experiments}

Our aim in this section is to numerically illustrate the convergence result stated in Theorem 4.1. To this end we used a truncated nonsmooth Newton multigrid (TNNMG-) method [12] to solve the capillarity problem. The implementation was done in C++ using the Distributed and Unified Numerics Environment (DUNE) [1].

The TNNMG-method consists of two half-steps, namely a nonlinear Gauss-Seidel step and a Newton correction. In the correction step a truncated approximate linearization is used and evaluated inexactly by a multigrid step. Numerical experiments usually display fast convergence due to the Newton step, which is suitably damped and projected in order to preserve the global convergence of the Gauss-Seidel method.

In [11] the TNNMG-method is introduced for quadratic obstacle problems. Its convergence is proven for a class of convex problems with nonlinearities which are smooth like the minimal surface term, decouple in the one-dimensional Euclidean directions like obstacles, or are a combination of these two types in [9] and [10]. In our case, the negative $-\kappa$ introduces a nonconvexity which is not covered by this theory. A generalization to problems where convexity holds only locally should be possible but is not the focus of this work.

We consider the discrete capillarity problem (13) for mesh sizes $h_{k}=\sqrt{2} 2^{-(k+1)}$ for $k=$ $0, \ldots, 8$ on a disc of diameter 1 with the parameters $-\kappa=-0.1, \beta=-0.8$, a prescribed volume $V=\pi$, and a constant obstacle at height 0 . A reference solution $u_{f}$ was computed with a mesh size of $h=\sqrt{2} 2^{-10}$. The graph of $u_{f}$ can be observed in Figure 2 .

The convergence result Theorem 4.1 essentially bounds the approximation error in the $W^{1,2}\left(\Omega_{h}\right)$-seminorm, and the full norm estimate comes from Poincaré's inequality. Since we want to observe the order of convergence we will monitor the errors $\left|u_{h}-u_{f}\right|_{0,2, \Omega_{h}}$ and $\left|u_{h}-u_{f}\right|_{1,2, \Omega_{h}}$ as functions of the mesh size parameter $h$.

The expected linear decay of the error in the $W^{1,2}$-seminorm can be observed in Figure 3. For the approximation error in the $L^{2}$-norm we observe quadratic decay. This corresponds to the well-known convergence behavior of minimal surfaces [21].

An essential ingredient in the proof of Theorem 4.1 is the $\kappa$-independence of the error. We tested this by repeating the above test for varying $\kappa$. The reference solution was computed with

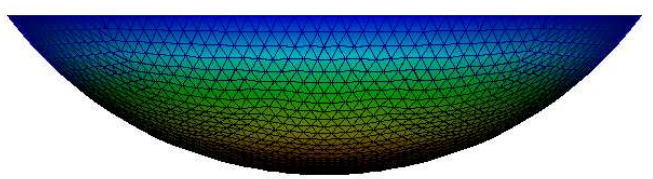

FIG. 2. Discrete capillary surface for $-\kappa=-0.1$ 


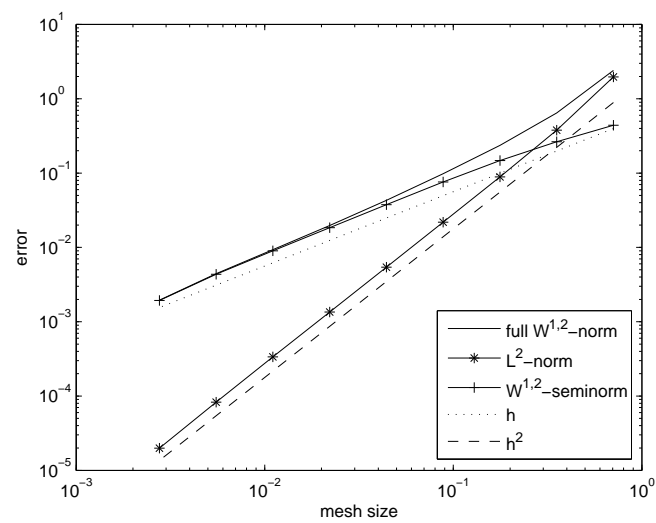

FIG. 3. Doubly logarithmic plot of the $W^{1,2}$-error over the mesh size $h$ for $-\kappa=-0.1$

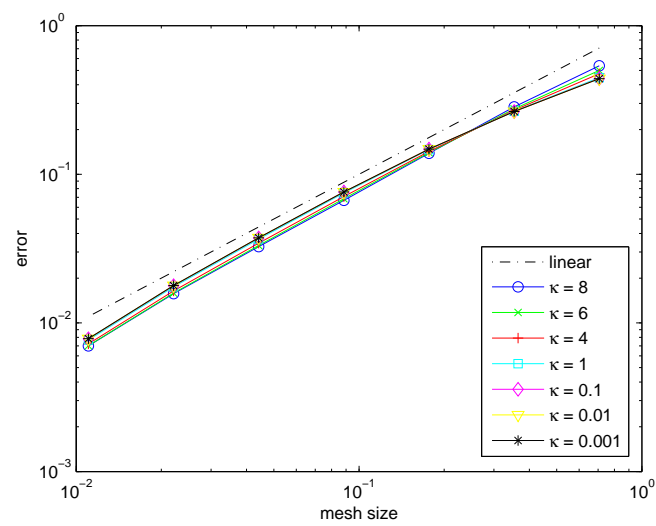

FIG. 4. Doubly logarithmic plot of the error in the $W^{1,2}$-seminorm over the mesh size $h$ for varying $\kappa$

$h=\sqrt{2} 2^{-8}$ and $h_{k}$ is as above with $k=0, \ldots, 6$. In the numerical results the discretization error indeed does not appear to depend on $\kappa$ as can be observed in Figure 4.

\section{REFERENCES}

1. Bastian, P., Blatt, M., Dedner, A., Engwer, C., Klöfkorn, R., Kornhuber, R., Ohlberger, M., \& SANDER, O. A generic grid interface for parallel and adaptive scientific computing. Part II: Implementation and tests in DUNE. Computing 82 (2008), 121-138. Zb11151. 65088 MR2421580

2. Ciarlet, P. The Finite Element Method for Elliptic Problems. North-Holland, 1978. Zb10383.65058 MR0520174

3. DzIUK, G. Finite elements for the Beltrami operator on arbitrary surfaces. In Partial Differential Equations and Calculus of Variations. Lecture Notes in Mathematics, S. Hildebrandt and R. Leis, Eds., vol. 1357. Springer, 1988, 142-155. Zb10663.65114 MR0976234 
4. Evans, L. Partial Differential Equations. AMS, 1998. Zb10902. 35002 MR1625845

5. FINN, R. Existence and non existence of capillary surfaces. Manuscripta Mathematica 28 (1979), 1-11. Zbl0421. 49043 MR0535691

6. Gerhardt, C. Existence and regularity of capillary surfaces. Bolletino U.M.I 10 (1974), 317-335. Zbl0314. 49019 MR0365316

7. Gerhardt, C. Global regularity of the solutions to the capillarity problem. Annali della Scuola Normale Superiore di Pisa 31 (1976), 157-175.

8. Gilbarg, D., \& Trudinger, N. Elliptic Partial Differential Equations of Second Order. Springer, 1998. Zbl1042.35002 MR1814364

9. GRÄSER, C. Convex minimization and phase field models. Phd thesis, Freie Universität Berlin, 2011.

10. GRÄSER, C. Truncated nonsmooth Newton multigrid methods for anisotropic convex minimization problems. Preprint, Matheon Berlin, 2013. in preparation.

11. Grëser, C., \& Kornhuber, R. Multigrid methods for obstacle problems. J. Comp. Math. 27 (2009), 1-44. Zb11199.65401 MR2493556

12. GRÄSER, C., SACK, U., \& SANDER, O. Truncated nonsmooth Newton multigrid methods for convex minimization problems. In Domain Decomposition Methods in Science and Engineering XVIII, vol. 70 of Lecture Notes in Computational Science and Engineering. Springer, 2009, 129-136. Zb11183.65076 MR2743965

13. Huisken, G. Capillary surfaces in negative gravitational fields. Mathematische Zeitschrift 185 (1984), 449-464.

14. Huisken, G. Capillary surfaces over obstacles. Pacific Journal of Mathematics 117 (1985), 121-141. Zbl0506. 49005 MR0733767

15. Johnson, C., \& ThoméE, V. Error estimates for a finite element approximation of a minimal surface. Mathematics of Computation 29130 (1975), 343-349. Zbl0302. 65086 MR0658321

16. Kinderlehrer, D., \& StAmpacchiA, G. An Introduction to Variational Inequalities and their Applications. Academic Press, 1980. Zb10457. 35001 MR0567696

17. Ladyzhenskaya, O., \& URAL'tseva, N. Linear and Quasilinear Elliptic Equations, vol. 46 of Mathematics in Science and Engineering. Academic Press, 1968. Zbl0164.13002 MR0244627

18. LaVerón-Simavilla, A., \& Perales, J. Equilibrium shapes of non-axisymmetric liquid bridges of arbitrary volume in gravitational fields and their potential energy. Physics of Fluids 7 (1995), 1204-1213. Zbl1027.76511

19. Mittelmann, H. On the approximation of capillary surfaces in a gravitational field. Computing 18 (1977), 141-148. Zbl0364.65101 MR0436779

20. NeČAs, J. Les Méthodes Directes en Théorie des Équations Elliptiques. Academia, 1967. Zbl1225.35003 MR0227584

21. Rannacher, R. Some asymptotic error estimates for finite element approximation of minimal surfaces. Rairo-Analyse numérique 11 (1977), 181-196. Zbl0356. 35034 MR0445866

22. Sumesh, P., \& Govindarajan, R. The possible equilibrium shapes of static pendant drops. Journal of chemical physics 133 (2010), 144707. 